\section{L'Actualité économique}

L'ACTUALITÉ ÉCONOMIQUE

Manpower and the Growth of Producer Services, par HARRY I. REVUE D'ANALYSE ÉCONOMIQUE

GREENFIELD. Un vol., 6/4 po. $\times$ 91/4 relié, 144 pages. COLUMBIA UNIVERSITY PRESS, New York et London, 1966. $(\$ 6.00)$

\title{
Denis Germain
}

Volume 43, numéro 4, janvier-mars 1968

URI : https://id.erudit.org/iderudit/1003105ar

DOI : https://doi.org/10.7202/1003105ar

Aller au sommaire du numéro

Éditeur(s)

HEC Montréal

ISSN

0001-771X (imprimé)

1710-3991 (numérique)

Découvrir la revue

Citer ce compte rendu

Germain, D. (1968). Compte rendu de [Manpower and the Growth of Producer Services, par HARRY I. GREENFIELD. Un vol., 61/4 po. x 91/4 relié, 144 pages. COLUMBIA UNIVERSITY PRESS, New York et London, 1966. (\$6.00)]. L'Actualité économique, 43(4), 782-783. https://doi.org/10.7202/1003105ar d'utilisation que vous pouvez consulter en ligne. 


\section{I'ACTUALITE ECONOMIQUE}

dans le « cercle vicieux $»$ de la pauvreté tandis que le Nord bénéficiait du dynamisme d'une région en expansion. Le maintien des disparités né s'explique que si nous raisonnons en termes dynamiques de processus cumulatifs.

La conclusion de l'ouvrage est que ce qu'il faut viser c'est à enrichir les régions pauvres et non pas à appauvrir les régions riches. Cette politique d'égalisation doit déboucher sur une amélioration générale dans l'ensemble de l'économie. C'est là une affirmation sur laquelle il y a beaucoup à dire et qui ne s'appuie sur aucune preuve. La politique du développement régional ne conduit pas automatiquement à une baisse du taux de croissance nationale. Mais elle peut y conduire et il convient au moins de se demander dans quelles conditions optimum régional et optimum national sont compatibles. Sur ce point les affirmations de lauteur sont plus du domaine de la morale que de la science économique. Nous reprochons d'ailleurs d'une façon générale à cet ouvrage de manquer de rigueur dans l'observation et l'analyse des faits. Les traitements statistiques en particulier sont rudimentaires et ne permettent pas d'aller très loin dans la compréhension du réel. Dans cés conditions on comprend que les recommandations de politique économique soient assez discutables. Cette situation est aggravée par l'insuffisance de supports théoriques de base : une bonne compréhension de l'expérience italienne aurait sans doute impliqué une recherche théorique approfondie et la connaissance d'autres expériences concrètes. Sur ces divers points l'apport de Saville est très inférieur à d'autres apports. En langue française nous pouvons par exemple citer la thèse de Bourguignat (Espaces économiques et intégration européenne) que Saville ne connaît pàs et qui correspond selon nous à une approche valable du problème.

R. Jouandet-Bernadat.

Manpower and the Growth of Producer Services, par HARRY I. GreENFIELD. Un vol., $61 / 4$ po. $x$ 91/4, relié, 144 pages. - Columbia UnIVERSITY PRESS, New York et London, 1966. (\$6.00).

Depuis plusieurs décennies déjà, l'importance du secteur des services par rapport à l'ensemble des activités économiques s'accroît constamment et à un rythme élevé. Ainsi, aux Etats-Unis, l'emploi, dans ce secteur, a aug. menté de 22.4 p.c. de r950 à 1960, comparé à 15.2 p.c. pour l'emploi total. En I960, 62.2 p.c. des travailleurs étaient dans le secteur des services et leurs revenus représentaient 59 p.c. du total.

Il existe une gamme très étendue de services ce qui oblige le chercheur, dans ce domaine, à les grouper selon certains critères, ces derniers étant fonction du but à atteindre. On peut distinguer, par exemple, deux grandes catégories de services. Ceux qui sont fournis aux individus et ceux qui sont fournis aux producteurs. Aux Etats:Unis, en 1960, les premiers employaient 49 p.c. de la main-d'œuvre totale et fournissaient 37 p.c. du revenu alors que les seconds, avec 13.1 p.c. de l'emploi total, fournissaient 22.7 p.c. du revenu total. 


\section{LES LIVRES}

Dans cette étude des services aux producteurs (producer services), monsieur Greenfield a dû faire face, au départ, au problème causé par le chevau. chement fréquent des deux grandes catégories de services déjà mentionnées. Par différentes méthodes, il est arrivé à isoler, dans une proportion satisfaisante pour son étude, les services rendus aux producteurs. Mentionnons entre autres : le transport, les communications, le commerce de gros, la finance, l'assurance et l'immeuble, le génie et l'architecture, la comptabilité, les services gouvernementaux. $\AA$ eux seuls, le transport, le commerce de gros ainsi que la finance, l'assurance et l'immeuble, représentaient, en 1960 , les deux tiers du total des emplois dans le secteur des services aux producteurs.

Les chapitres qui suivent ont trait à l'offre et à la demande de ce type de services, à la main-d'œuvre utilisée, à leur répartition régionale et industrielle, à leur rôle dans la croissance économique.

L'auteur arrive à la conclusion que les services aux producteurs jouent un rôle qui n'est pas négligeable dans la croissance économique. Cependant, la mesure du phénomène présente des difficultés importantes à cause du peu d'attention qu'on y a porté jusqu'à maintenant. En conclusion, il mentionne rapidement les conséquences de ce phénomène sur la politique de la main. d'œuvre et sur la politique économique dans son ensemble. Il propose aussi quelques sujets de recherche capables de jeter plus de lumière sur ce secteur de l'économie.

Denis Germain

\section{NOTES BIBLIOGRAPHIQUES}

Les régimes de retraite, par JoSEPH FlesCh. Un vol., $4 \frac{1}{2}$ po. $x$ 7, broché, 128 pages. Collection \& Que sais-je ? . - Presses Universitaires De France, 108, boul. St-Germain, Paris, 1967. (3 F.).

Depuis le début du siècle, l'espérance de vie à la naissance est passée de 45 à 66 ans pour les hommes et de 48 à 72 ans pour les femmes. Lespoir d'atteindre lâge de la retraite est donc de plus en plus fondé. Mais les problèmes psychologiques, la perte du revenu professionnel, le déclin pathologique de la vieillesse sont de nouvelles difficultés qui assaillent les hommes au seuil de cette nouvelle étape. Des systèmes collectifs sont nés et sont considérés aujourd'hui comme une nécessité vitale et comme la reconnaissance d'un droit. Le retraité n'est plus une charge, mais un créancier de la société. Les pensions de vieillesse sont devenues des « revenus professionnels différés ». Cette tendance est inscrite dans l'évolution des régimes de retraite 